\title{
Elevated brain lesion volumes in older adults who use calcium supplements: a cross-sectional clinical observational study
}

\author{
Martha E. Payne ${ }^{1 *}$, Douglas R. McQuoid ${ }^{1}$, David C. Steffens ${ }^{2}$ and John J. B. Anderson ${ }^{3}$ \\ ${ }^{1}$ Department of Psychiatry and Behavioral Sciences, Duke University, 2200 West Main Street, Suite B210, \\ Durham, NC 27705, USA \\ ${ }^{2}$ Department of Psychiatry, University of Connecticut Health Center, Farmington, CT, USA \\ ${ }^{3}$ Department of Nutrition, Gillings School of Global Public Health, University of North Carolina, Chapel Hill, NC, USA \\ (Submitted 8 August 2013 - Final revision received 21 February 2014 - Accepted 5 March 2014 - First published online 1 May 2014)
}

\begin{abstract}
Recent studies have implicated Ca supplements in vascular risk elevation, and therefore these supplements may also be associated with the occurrence of brain lesions (or hyperintensities) in older adults. These lesions represent damage to brain tissue that is caused by ischaemia. In the present cross-sectional clinical observational study, the association between Ca-containing dietary supplement use and lesion volumes was investigated in a sample of 227 older adults (60 years and above). Food and supplemental Ca intakes were assessed with the Block 1998 FFQ; participants with supplemental Ca intake above zero were categorised as supplement users. Lesion volumes were determined from cranial MRI ( 1.5 tesla) scans using a semi-automated technique; volumes were log-transformed because they were non-normal. ANCOVA models revealed that supplement users had greater lesion volumes than non-users, even after controlling for food $\mathrm{Ca}$ intake, age, sex, race, years of education, energy intake, depression and hypertension (Ca supplement use: $\beta=0 \cdot 34$, sE $0 \cdot 10$, $\left.F_{1,217}=10 \cdot 98, P=0 \cdot 0011\right)$. The influence of supplemental $\mathrm{Ca}$ use on lesion volume was of a magnitude similar to that of the influence of hypertension, a well-established risk factor for lesions. Among the supplement users, the amount of supplemental Ca was not associated with lesion volume $\left(\beta=-0.000035\right.$, se $\left.0.00015, F_{1,139}=0 \cdot 06, P=0 \cdot 81\right)$. The present study demonstrates that the use of Ca-containing dietary supplements, even low-dose supplements, by older adults may be associated with greater lesion volumes. Evaluation of randomised controlled trials is warranted to determine whether this relationship is a causal one.
\end{abstract}

Key words: Calcium supplements: Brain lesions: MRI hyperintensities: Older adults

Brain lesions, also known as hyperintensities, are areas of damage observed on brain MRI (Fig. 1). These lesions are common in older adults and increase the risk of devastating health outcomes, including depression, cognitive decline, dementia, stroke, physical disability, hip fracture and death ${ }^{(1-10)}$. Postmortem studies have determined that these lesions form primarily due to ischaemia, especially larger lesions $(>3 \mathrm{~mm})$ and lesions found in depressed individuals ${ }^{(11)}$.

In our previous study, we had found higher total Ca intakes to be associated with greater brain lesion volumes in older adults, indicating Ca's potential as an aetiological factor for lesions $^{(12)}$. Given that lesions primarily result from ischaemic damage, this finding on $\mathrm{Ca}-$ lesion relationship is consistent with recent reports showing that excess $\mathrm{Ca}$, especially that from dietary supplements, may increase one's risk of cardiovascular outcomes, including myocardial infarction, incident CHD, stroke and IHD deaths ${ }^{(13-18)}$. For example, in the Heidelberg cohort ( $n$ 23980), Ca supplement use was found to significantly increase the risk of myocardial infarction over 11 years of follow-up (hazard ratio 1.86) ${ }^{(19)}$. Supplemental Ca may be particularly harmful because of its effect on serum Ca concentrations ${ }^{(20,21)}$. Serum concentrations even within the normal range have been shown to be associated with arterial calcification ${ }^{(22,23)}$ and other cardiovascular pathologies $^{(24)}$. It is important to determine the contribution of supplemental Ca to lesions, given the detrimental effects of lesions and the modifiable nature of $\mathrm{Ca}$ intake. This is especially relevant for women's health because they are greater consumers of $\mathrm{Ca}$ supplements, primarily due to concerns about osteoporosis ${ }^{(25)}$. In addition, women are at a greater risk of depression and dementia, conditions that are partially caused by ischaemic brain lesions ${ }^{(1,5)}$

The relative importance of Ca supplements, as opposed to food $\mathrm{Ca}$, was not established in our previous study on $\mathrm{Ca}$-lesion relationship in a sample of older adults. Therefore, the aim of the present study was to evaluate differences in

Abbreviations: DDES, Duke Depression Evaluation Schedule; NIRL, Neuropsychiatric Imaging Research Laboratory.

*Corresponding author: Dr. M. E. Payne, fax +1919416 7547, email martha.payne@duke.edu 

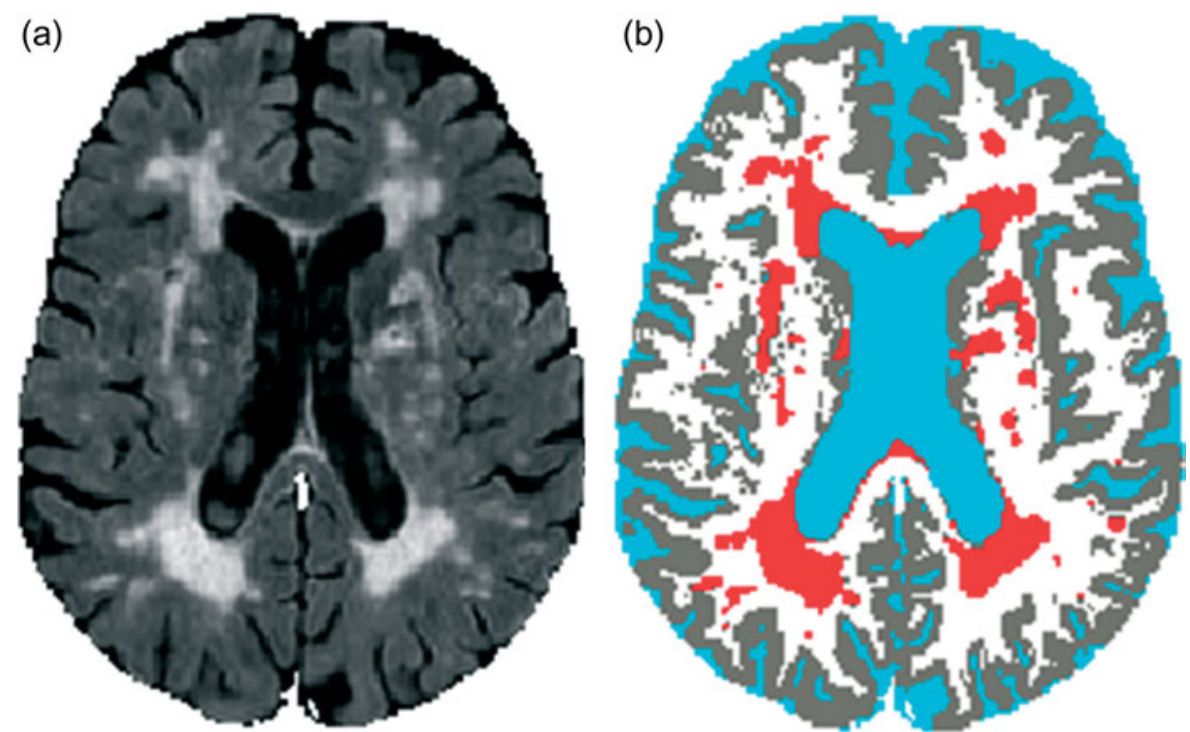

Fig. 1. Brain lesions observed on MRI: fluid-attenuated inversion recovery image (a) and tissue classification image (b) (lesions in red).

lesion volumes between users and non-users of Ca-containing dietary supplements in the same study sample while also exploring the influence of supplement dose and duration, as well as sex differences and vitamin D. We hypothesised that supplement users would have greater lesion volumes than non-users, irrespective of the dietary $\mathrm{Ca}$ intake.

\section{Methods}

The present cross-sectional clinical study was carried out during a larger longitudinal cohort examination of depressed and non-depressed older adults (NeuroCognitive Outcomes of Depression in the Elderly), which began in 1994 and had enrolment going on until $2011^{(26)}$. Dietary intake data were collected annually beginning in 1999. MRI brain scans began to be taken in 1994 and were acquired every 2 years. In the present study, data obtained from only one MRI scan and one dietary intake assessment were used for each participant. In analyses, lesion volumes determined from the MRI scan taken at a time point closest to that of the first available dietary intake assessment were used.

The present study was conducted according to the guidelines laid down in the Declaration of Helsinki, and all procedures involving human subjects were approved by the Duke University Institutional Review Board, Durham, North Carolina, USA. Written informed consent was obtained from all participants before the start of the study.

\section{Sample}

All participants who had both dietary intake data (from the FFQ) and MRI lesion volume data were included in the present study ( $n$ 227). Only those with scans acquired with a 1.5-tesla scanner were included in the study. The study sample included individuals who met diagnostic criteria for major depressive disorders (according to the Diagnostic and Statistical Manual of Mental Disorders, Fourth Edition ${ }^{(27)}$ ) at study baseline and never-depressed comparison participants recruited from the community. Participants were aged 60 years or above and were able to speak and write in English.

Exclusion criteria included a concurrent diagnosis of a psychiatric or neurological illness and significant cognitive impairment (as indicated by a Mini-Mental State Examination ${ }^{(28)}$ score of less than 24 out of 30). In addition, participants with severe depression symptomatology were excluded because of concerns about (1) subject burden, given the duration of FFQ administration and difficulty in filling it up and (2) memory difficulty, common in severe depression, which could affect the accuracy of reporting. This criterion was evaluated by the treating psychiatrist on a case-by-case basis, an approach used because that clinician was most familiar with a patient's limitations. Comparison participants (controls) were required to have no evidence of a depression diagnosis or lifetime history of depression.

\section{Treatment}

Depressed participants received individualised treatment from a psychiatrist, who followed them up throughout the study. Most depressed participants received antidepressant medication.

\section{Nutrition assessment}

The dietary intake protocol has been described previously ${ }^{(29)}$. The 1998 Block FFQ (NutritionQuest) was self-administered to assess dietary intake over the previous year. For approximately $90 \%$ of the participants, the dietary intake assessment year either preceded or overlapped with the MRI scan acquisition year. The 1998 Block is an updated version of the FFQ developed by Gladys Block at the National Cancer Institute ${ }^{(30)}$ and has been validated against other dietary intake assessment instruments ${ }^{(31)}$. Returned questionnaires were checked for completeness and excluded if questions on more than fifteen 
food items were skipped. The 1998 version of the Block FFQ included duration and frequency items on the use of dietary supplements, including Ca supplements and mineral-containing multivitamins. Intake estimates included total energy $(\mathrm{kJ})$, $\mathrm{Ca}(\mathrm{mg})$ and vitamin D $(\mu \mathrm{g})$. Ca and vitamin D intakes were calculated from both foods and dietary supplements. Participants with supplemental $\mathrm{Ca}$ intake greater than $0 \mathrm{mg} / \mathrm{d}$ were categorised as $\mathrm{Ca}$ supplement users, while those with intake equal to $0 \mathrm{mg} / \mathrm{d}$ were categorised as non-users. The duration of supplement use included six categories (less than 1 year, 1 year, 2 years, 3-4 years, 5-9 years, and 10 or more years).

\section{Lesion volumes}

The participants were imaged with a 1.5-tesla whole-body MRI system (Signa; GE Medical Systems) under an Institutional Review Board-approved protocol. The pulse sequence parameters have been described previously ${ }^{(32)}$. The magnetic resonance images were processed for lesion volumes by blinded (to all identifying information, including depression diagnosis and dietary intake) analysts in the Neuropsychiatric Imaging Research Laboratory (NIRL).

A dual-echo fast spin-echo axial acquisition was used for the volumetric measurement of brain structures, including grey and white matter lesions. The NIRL image processing procedure has been described previously ${ }^{(32)}$. The procedure is a supervised, semi-automated method that uses the multiple magnetic resonance contrasts available to identify different tissue classifications through a 'seeding' process wherein a trained analyst manually selects pixels in each tissue type that is to be identified (such as grey matter, white matter, cerebrospinal fluid, lesions and background). Grey and white matter lesion areas were then selected based on a set of rules that allow trained analysts to reliably select lesion regions. Periventricular lesions were defined as regions that were contiguous with the lateral ventricles and did not extend into the white matter tracts. These lesions were classified as white matter lesions. Deep white matter lesions were located in the white matter tracts and may or may not have adjoined periventricular lesions. Subcortical grey matter lesions were defined as lesions within the basal ganglia or thalamus. Lesion volumes were derived by multiplying the lesion area on each slice by the slice thickness $(3 \mathrm{~mm})$ and then summing up lesion volumes from all slices. Total lesion volume included both grey matter lesion volume and white matter lesion volume, although white matter lesion volume predominated. For purposes of the present study, only total lesion volume was examined.

\section{Statistical analyses}

All statistical analyses were carried out using the SAS software, version 9.3 TS (serial no. 70007112; SAS Institute). Significance was defined at $P<0.05$ and higher significance was defined at $P<0 \cdot 01$.

Covariates of interest were demographic variables (age, sex and race), years of education, group (depression $v$. comparison) and hypertension. Hypertension ('high blood pressure') was included with self-reported physical health items from the National Institute of Mental Health Diagnostic Interview Schedule ${ }^{(33)}$ portion of the Duke Depression Evaluation Schedule (DDES), which was administered annually. BMI $\left(\mathrm{kg} / \mathrm{m}^{2}\right)$ was calculated from self-reported weight and height at the time of FFQ administration.

In statistical analyses, lesion volumes obtained from the MRI scan taken at a time point closest to that of the dietary intake assessment were used. For most participants, the MRI and dietary intake assessments were separated by an interval of less than 1 year. To minimise the time interval, either assessment could precede the other. Self-reports of hypertension were obtained from the closest annual DDES instrument. Given the relatively small numbers of minority participants, race was dichotomised as White $v$. non-White. As lesion volumes were non-normal, values were log-transformed before being used in statistical models (LogLesion).

For bivariate analysis, $t$ tests were carried out on continuous level variables and $\chi^{2}$ tests were carried out on categorical variables testing for differences between Ca supplement users and non-users. ANCOVA models were fit to test differences between supplement users and non-users upon lesion volume while controlling for covariates. Covariates included age, sex, race, years of education, hypertension, depression, total energy intake and food Ca intake. BMI was omitted from the models because it was not significant in the previous $\mathrm{Ca}$-lesion study ${ }^{(12)}$. In secondary analyses, the main ANCOVA model was used to examine the potential for the occurrence of sex differences (addition of interaction term (sex $\times$ supplement use)), as well as influence of vitamin $\mathrm{D}$ intake (inclusion of total vitamin $\mathrm{D}$ intake).

The approximate power of the main model was 0.908 (based on a type III $F$ test for multiple regression using PROC POWER considering nine predictors and 227 observations, controlling for a type I error of 0.05).

\section{Results}

\section{Participant characteristics}

A total of 285 participants had a 1.5-tesla MRI scan and were asked to complete a FFQ. Of these, 227 participants completed the FFQ. A comparison of the FFQ responders and non-responders revealed no differences in age, sex, race, years of education, hypertension or lesion volume, but non-responders were found to more likely be in the depression group ( $n 45$ depressed $v \cdot n 13$ non-depressed; $\left.\chi^{2} 23 \cdot 02, \mathrm{df}=1, P<0 \cdot 0001\right)$. The sample of the present study ( $n$ 227) was very similar to that of the previous study $(n 232)^{(12)}$; small differences were due to the inclusion of new participants, as well as the removal of data of a few participants from the study archive, which was done after the completion of the previous study.

Of the 227 older adult participants (see Table 1), 149 were categorised as users of Ca-containing dietary supplements (range of daily supplemental intake: $37-1130 \mathrm{mg}$ ) and seventy-eight as non-users (i.e. supplemental intake: $0 \mathrm{mg}$ ). Supplement users were found to more likely be females $\left(\chi^{2} 14 \cdot 22, \mathrm{df}=1, P=0.0002\right)$ and have more years of education $(t=-3.04, \mathrm{df}=128.96, P=0.0029)$ compared with non-users. Groups also differed by race, as White participants 
Table 1. Sociodemographic, co-morbidity, lesion and diet characteristics of the study participants

(Mean values with their standard errors; number of participants and percentages)

\begin{tabular}{|c|c|c|c|c|c|c|c|}
\hline & \multicolumn{2}{|c|}{ Total ( $n$ 227) } & \multicolumn{2}{|c|}{$\begin{array}{l}\text { Ca supplement users } \\
\qquad(n 149)\end{array}$} & \multicolumn{2}{|c|}{ Non-users $(n 78)$} & \multirow[b]{2}{*}{$P^{\star}$} \\
\hline & Mean & SE & Mean & SE & Mean & SE & \\
\hline Age (years) & $70 \cdot 9$ & 0.4 & $71 \cdot 2$ & 0.5 & $70 \cdot 3$ & 0.7 & 0.3 \\
\hline Sex (female) & & & & & & & 0.0002 \\
\hline$n$ & \multicolumn{2}{|c|}{148} & \multicolumn{2}{|c|}{110} & \multicolumn{2}{|c|}{38} & \\
\hline$\%$ & \multicolumn{2}{|c|}{65} & \multicolumn{2}{|c|}{74} & \multicolumn{2}{|c|}{49} & \\
\hline Race & & & & & & & 0.02 \\
\hline \multicolumn{8}{|l|}{ White } \\
\hline$n$ & \multicolumn{2}{|c|}{193} & \multicolumn{2}{|c|}{133} & \multicolumn{2}{|c|}{60} & \\
\hline$\%$ & \multicolumn{2}{|c|}{85} & \multirow{2}{*}{\multicolumn{2}{|c|}{89}} & \multicolumn{2}{|c|}{77} & \\
\hline \multicolumn{7}{|l|}{ African-American } & \\
\hline$n$ & \multirow{2}{*}{\multicolumn{2}{|c|}{$\begin{array}{l}22 \\
10\end{array}$}} & \multicolumn{2}{|c|}{8} & \multicolumn{2}{|c|}{14} & \\
\hline$\%$ & & & & & & & \\
\hline \multicolumn{8}{|l|}{ Asian } \\
\hline$n$ & \multicolumn{2}{|c|}{5} & \multicolumn{2}{|c|}{4} & \multicolumn{2}{|c|}{1} & \\
\hline$\%$ & \multicolumn{2}{|c|}{2} & \multicolumn{2}{|c|}{3} & & & \\
\hline Multiracial & & & & & & & \\
\hline$n$ & & & & & & & \\
\hline$\%$ & & & & & & & \\
\hline Education (years) & $14 \cdot 8$ & 0.2 & $15 \cdot 1$ & 0.2 & $14 \cdot 1$ & 0.3 & 0.003 \\
\hline Hypertension (yes) & & & & & & & 0.5 \\
\hline$n$ & & & & & & & \\
\hline$\%$ & & & & & & & \\
\hline Depression (yes) & & & & & & & 0.05 \\
\hline$n$ & & & & & & & \\
\hline$\%$ & & & & & & & \\
\hline $\mathrm{Ca}(\mathrm{mg} / \mathrm{d})$ & & & & & & & \\
\hline Food & 797.0 & $27 \cdot 0$ & $848 \cdot 8$ & 34.4 & 698.0 & $40 \cdot 7$ & 0.008 \\
\hline Supplemental & 488.5 & 33.3 & 744.2 & $36 \cdot 0$ & 0 & NA & NA \\
\hline Total & 1285.5 & $46 \cdot 4$ & 1593.0 & 51.9 & 698.0 & $40 \cdot 7$ & $<0.0001$ \\
\hline Vitamin $D(\mu \mathrm{g} / \mathrm{d}) \dagger$ & 8.6 & 0.4 & 11.3 & 0.4 & 3.5 & 0.4 & $<0.0001$ \\
\hline Energy $(\mathrm{kJ} / \mathrm{d})$ & $7227 \cdot 1$ & $189 \cdot 6$ & $7197 \cdot 8$ & $258 \cdot 0$ & 7283.1 & $250 \cdot 3$ & 0.8 \\
\hline BMI $\left(\mathrm{kg} / \mathrm{m}^{2}\right)$ & $26 \cdot 2$ & 0.3 & 25.9 & 0.4 & $26 \cdot 7$ & 0.5 & 0.2 \\
\hline Lesion volume (ml) & $6 \cdot 2$ & 0.5 & $7 \cdot 2$ & 0.8 & 4.1 & 0.4 & 0.0004 \\
\hline LogLesion & 1.4 & 0.05 & 1.5 & 0.07 & 1.2 & 0.07 & 0.0005 \\
\hline Geometric mean $(\mathrm{ml}) \S$ & $4 \cdot 1$ & $1 \cdot 1$ & 4.6 & $1 \cdot 1$ & $3 \cdot 3$ & $1 \cdot 1$ & NA \\
\hline
\end{tabular}

NA, not available.

${ }^{*} P$ value for difference between the groups ( $\chi^{2}$ test was used to compare proportions; $t$ test was used to compare means).

†Total intake (food + supplemental).

$\ddagger$ Natural logarithm of lesion volume.

$\S$ Geometric mean $=\mathrm{e}^{\text {(mean of LogLesion) }}$.

were found to more likely be supplement users, while nonWhites were found to more likely be non-users ( $\chi^{2} \quad 6 \cdot 12$, $\mathrm{df}=1, P=0 \cdot 01)$. In addition to higher intake of supplemental $\mathrm{Ca}$, users had significantly higher intake of food and beverage $\mathrm{Ca}(t=-2.69, \quad \mathrm{df}=225, \quad P=0.0076)$, total $\mathrm{Ca} \quad(t=-13.56$, $\mathrm{df}=223.53, \quad P<0.0001)$ and total vitamin $\mathrm{D} \quad(t=-13.97$, $\mathrm{df}=221 \cdot 19, P<0 \cdot 0001)$ than non-users. Users had significantly greater lesion volumes $(t=-3.63, \mathrm{df}=209 \cdot 5, P=0.0004)$ than non-users. Geometric mean for lesion volume is included in Table 1 as a measure of central tendency that is less prone to distortion by outliers and may also be easier to interpret than logarithmic values, given that geometric means have the same units as the original variable (i.e. $\mathrm{ml}$ for lesion volume).

\section{ANCOVA models}

To examine the effect of Ca supplement use (yes/no) on LogLesion while controlling for potential confounders, we used
ANCOVA models. These models revealed that users of Ca-containing supplements had significantly higher LogLesion values than non-users, after controlling for dietary Ca intake (food Ca), age, sex, race, years of education, energy intake, depression and hypertension (Table 2). Significant covariates in the models were age, hypertension and depression. Food Ca intake was not significant in the models. Table 3 summarises lesion volume (predicted geometric mean) ratios between the groups for each of the significant categorical predictor variables, based on the full multivariable model. Ca supplement users had 1.4 times greater lesion volume than non-users. Among the users of Ca-containing supplements, the amount of supplemental $\mathrm{Ca}$ (mg) was not significantly associated with LogLesion $\left(\beta=-0.000035\right.$, SE $\left.0.00015, F_{1,139}=0.06, P=0.81\right)$ and nor was the duration of supplement use $(\beta=-0.040$, SE $0 \cdot 043$, $\left.F_{1,134}=-0.93, P=0.35\right)$. It should be noted that duration data were available for only 106 of the 149 participants, and no sex differences were found for the duration of supplement use. 
Table 2. Influence of supplemental calcium use on lesion volumes (a multivariable model with eight covariates)

\begin{tabular}{llllc}
\hline & Estimate & \multicolumn{1}{c}{ SE } & $F^{*}$ & $P$ \\
\hline Supplemental Ca (yes/no)† & 0.34 & 0.10 & 10.98 & 0.0011 \\
Food Ca (mg/d) & 0.00020 & 0.00014 & 2.02 & 0.16 \\
Age (years) & 0.061 & 0.0076 & 65.04 & $<0.0001$ \\
Sex (female/male) & 0.052 & 0.10 & 0.27 & 0.61 \\
Race (White/non-White) & 0.067 & 0.13 & 0.26 & 0.61 \\
Education (years) & 0.0057 & 0.021 & 0.07 & 0.79 \\
Hypertension (yes/no)ł & 0.27 & 0.099 & 7.62 & 0.0063 \\
Depression (yes/no)§ & 0.28 & 0.096 & 8.75 & 0.0034 \\
Energy (kJ/d) & 0.000010 & 0.000020 & 0.26 & 0.61 \\
\hline
\end{tabular}

*ANCOVA: df $F_{1,217}$ for all variables.

$\dagger$ Yes if supplemental calcium intake is greater than $0 \mathrm{mg} / \mathrm{d}$.

$\ddagger$ Determined from the closest annual self-report within the National Institute of Mental Health Diagnostic Interview Schedule portion of the Duke Depression Evaluation Schedule ${ }^{(33)}$.

$\S$ Yes if met diagnostic criteria for major depressive disorders according to the Diagnostic and Statistical Manual of Mental Disorders, Fourth Edition at study baseline ${ }^{(27)}$.

\section{Secondary analyses}

Male-female differences. A sex $\times$ supplement use interaction term was added to the main model, described previously, to examine the potential for the occurrence of sex differences in the relationship between Ca supplement use and LogLesion. This interaction term was not significant $\left(\beta=0 \cdot 27\right.$, sE $\left.0 \cdot 20, F_{1,216}=1 \cdot 80, P=0 \cdot 18\right)$.

Vitamin $D$ intake. When total vitamin $\mathrm{D}$ intake was added to the main model, Ca supplement use remained significantly associated with LogLesion (Ca supplement use: $\beta=0 \cdot 27$, SE $\left.0 \cdot 13, F_{1,216}=4 \cdot 29, P=0 \cdot 040\right)$. Vitamin $\mathrm{D}$ intake was not significantly associated with LogLesion in this model $\left(\beta=0.0092\right.$, se $\left.0.011, F_{1,216}=0 \cdot 71, P=0 \cdot 40\right)$.

\section{Discussion}

The main finding of the present study was that older adults who used Ca-containing dietary supplements had greater brain lesion volumes than non-users. This association was significant even after controlling for dietary Ca intake (from foods and beverages), as well as age, sex, race, years of education, energy intake, depression and hypertension. Given that dietary $\mathrm{Ca}$ intake was included in the main model and was found to be non-significant, supplemental Ca per se may be an important risk factor. The present study did not find sex differences in this relationship or a significant influence of vitamin D on the Ca-lesion relationship. Surprisingly, the duration of supplement use was not significantly associated with lesions, although duration data were available for only $71 \%$ of the users. It is possible that duration was confounded by co-morbid illness, a factor associated with lesion aetiology. Supplemental Ca amount was also not associated with lesion volume, among the supplement users, indicating that the use of any Ca-containing dietary supplement, regardless of the dose, may lead to risk.

The use of Ca-containing dietary supplements has not been investigated previously in relationship with brain lesions. However, several randomised controlled trials have examined the role of Ca supplements in the risk of stroke, which may be relevant to the present findings, given that lesions and stroke often have a shared aetiology. A recent meta-analysis has examined data obtained from 28072 randomised controlled trial participants, of whom 768 individuals suffered an incident stroke, and shown a trend for an increased risk of stroke among those assigned to receive a $\mathrm{Ca}$ supplement (relative risk $1 \cdot 15$, CI $1.00,1.32 ; P=0.06)^{(17)}$. A re-analysis of the Women's Health Initiative Calcium-Vitamin D randomised controlled trial revealed that Ca supplementation resulted in an increased cardiovascular risk only in those participants who were not taking personal Ca supplements at the time of randomisation and also showed that the dose of Ca supplement was not related to risk $^{(17)}$. The researchers suggested that these findings indicate the lack of a dose-response relationship for $\mathrm{Ca}$ and vascular risk and that even low-dose supplementation may result in risk. This notion is consistent with the results of the present study, for which supplement use was defined as the use of any Ca-containing supplement, including multivitamin/mineral supplements, which generally have much smaller doses of Ca (e.g. 100-200 mg/pill) than Ca-only or Ca plus vitamin D preparations. Among the supplement users, the total amount of supplemental Ca was not associated with lesion volume, consistent with the Bolland analysis $^{(17)}$. It is possible that even small-dose supplements may be harmful, perhaps by stressing the Ca homeostatic mechanism. Alternatively, the use of Ca-containing supplements may be associated with another factor associated with brain lesions.

Large quantities of $\mathrm{Ca}$ found in a single dose of a supplement, often referred to as loading, have been found

Table 3. Geometric means of lesion volume by calcium supplement use, depression and hypertension*

\begin{tabular}{|c|c|c|c|c|}
\hline $\begin{array}{l}\text { Predictor } \\
\text { condition }\end{array}$ & Yes & No & $\begin{array}{c}\text { Ratio of geometric } \\
\text { means of lesion } \\
\text { volume (yes:no)† }\end{array}$ & $\mathrm{Cl}$ \\
\hline $\begin{array}{l}\text { Ca supplement } \\
\text { use }\end{array}$ & $5.07 \mathrm{ml}$ & $3.63 \mathrm{ml}$ & 1.40 & $1.15,1.71$ \\
\hline Depression & $4.94 \mathrm{ml}$ & $3.72 \mathrm{ml}$ & 1.32 & $1 \cdot 10,1 \cdot 61$ \\
\hline Hypertension & $4.92 \mathrm{ml}$ & $3.74 \mathrm{ml}$ & $1 \cdot 31$ & $1.08,1.60$ \\
\hline
\end{tabular}


to cause a substantial and rapid increase in serum Ca concentrations, proportional to Ca supplement load ${ }^{(20)}$, which in turn may promote the development of $\mathrm{Ca}$ deposits in the vasculature (i.e. arterial calcification), primarily at sites of atheromas (fatty deposits). This calcification, in turn, may lead downstream to ischaemia and the development of brain lesions ${ }^{(34,35)}$. In addition to a vascular mechanism, excess $\mathrm{Ca}$ may have direct effects on brain health ${ }^{(36)}$, especially if there is damage to the blood-brain barrier, such as that occurring with advancing age or neurodegenerative conditions.

The primary modifiable risk factor that has previously been established for lesions is hypertension ${ }^{(37,38)}$. New data from the present study indicate that the effect of Ca supplements on lesions is similar in magnitude to that of hypertension (see Table 3). If this finding is confirmed in longitudinal studies, it could have important health implications - because it is obviously much easier to cease Ca supplement use than to medically manage hypertension. Age and depression were also significantly associated with lesions, consistent with prior reports ${ }^{(39,40)}$, while sex, race, years of education, energy intake and BMI were not significantly associated with lesions. The role of vitamin D in the development of brain lesions remains unclear. In our previous study, higher vitamin D intake was found to be significantly associated with greater lesion volumes ${ }^{(12)}$. However, a study that examined serum concentrations of vitamin $\mathrm{D}$ has found that vitamin $\mathrm{D}$ insufficiency, not supplementation or excess, is associated with greater lesion volumes ${ }^{(41)}$. It is possible that both excess intake and vitamin $\mathrm{D}$ insufficiency may promote lesions. Secondary analyses carried out in the present study revealed that the inclusion of total vitamin $\mathrm{D}$ intake in the main model did not eliminate the significant association between Ca supplement use and lesions, and vitamin D intake was not significant in the model. The interpretation of these results is complicated, given the generally high correlation between Ca supplement use and total vitamin $\mathrm{D}$ intake, lack of information on sun exposure, and the fact that many Ca supplements contain vitamin D. It is also unclear whether vitamin D intake would have a significant impact on a Ca pathology, given that the conversion of 25-hydroxyvitamin $\mathrm{D}$ to 1,25-dihydroxyvitamin $\mathrm{D}$ (the active hormone) might be limited at the medium-to-high $\mathrm{Ca}$ intakes reported by both groups in the present study ( $700 \mathrm{mg}$ and above).

The present study has a number of strengths, including the use of a highly reliable method for quantifying lesion volumes from MRI scans ${ }^{(32)}$, use of blinded imaging analysts and the relatively substantial sample size for a MRI study. The assessment of $\mathrm{Ca}$ intake using the validated Block FFQ is a further strength of the study. In addition, the models incorporated an extensive list of potential confounders, including hypertension and depression. The limitations of the study include its cross-sectional nature, which precludes the establishment of a causal relationship between Ca supplement use and lesion development, potential for the occurrence of residual confounding, and the lack of measures of serum $\mathrm{Ca}$, arterial calcification or other potential mediators. The objective measures of cardiometabolic risk, including glycaemic control and blood pressure, were unavailable. In addition, the Block
1998 assessment does not distinguish between Ca-only and combined $\mathrm{Ca} /$ vitamin D supplements and nor is Ca-containing antacid use specifically queried. Generalisation to older population groups may be limited, given that the study sample was from a clinical psychiatric study of older adults.

As the present study is the first to detect a positive relationship between supplemental Ca use and MRI-defined brain lesions, many questions remain to be answered. This potentially important finding needs to be replicated in samples of older adults that are more representative of the general population than the clinical psychiatric sample of the present study. In addition, temporality needs to be determined before causality between supplemental $\mathrm{Ca}$ use and brain lesions is established. Lastly, if supplemental Ca is determined to be causal for brain lesions, biochemical mechanisms need to be identified.

\section{Conclusion}

The use of Ca-containing dietary supplements by older adults was found to be associated with greater brain lesion volumes, even after controlling for the usual amount of dietary $\mathrm{Ca}$ intake. Interestingly, neither the amount of supplemental Ca nor the duration of supplemental Ca use was associated with lesion volume. These findings indicate that adverse biochemical effects of supplemental Ca use may exist in older adults, regardless of the dose. These results should be considered preliminary, given the cross-sectional design, potential for the occurrence of residual confounding and specialised study population. Evaluation of randomised controlled trials is warranted to examine the potential for the occurrence for an aetiological relationship between $\mathrm{Ca}$ supplement use and lesion development.

\section{Acknowledgements}

The authors thank the study participants for their dedication to furthering the knowledge of nutrition and brain health. They also thank Cortnee Pierce for subject recruitment, Robert Rybczynski for nutrition assessment assistance, Andrew Shiloh for data management, Celia Hybels for statistical guidance, Brian Boyd for lesion figure preparation and Scott Davis for editorial assistance. In addition, the authors appreciate the guidance of Connie Bales and Gerda Fillenbaum, who serve as mentors for the Building Interdisciplinary Research Careers in Women's Health (BIRCWH) K12 programme.

The present study was funded by the following United States National Institutes of Health grants: BIRCWH K12HD043446 (M. E. P.) and R01-MH054846 and K24-MH070027 (D. C. S.). The funding source had no role in the design and analysis of the study or in the writing of this article.

The authors' contributions are as follows: M. E. P. designed the study, interpreted the data and drafted the manuscript; D. R. M. analysed the data; M. E. P., D. R. M., D. C. S. and J. J. B. A. contributed to the interpretation of the data and revision of the manuscript; M. E. P. and D. C. S. financed the study.

None of the authors has any conflicts of interest to declare. 


\section{References}

1. Godin O, Dufouil C, Maillard P, et al. (2008) White matter lesions as a predictor of depression in the elderly: the 3CDijon study. Biol Psychiatry 63, 663-669.

2. Steffens DC, Krishnan KR, Crump C, et al. (2002) Cerebrovascular disease and evolution of depressive symptoms in the Cardiovascular Health Study. Stroke 33, 1636-1644.

3. de Groot JC, de Leeuw FE, Oudkerk M, et al. (2002) Periventricular cerebral white matter lesions predict rate of cognitive decline. Ann Neurol 52, 335-341.

4. van den Heuvel DM, Ten Dam VH, de Craen AJ, et al. (2006) Increase in periventricular white matter hyperintensities parallels decline in mental processing speed in a non-demented elderly population. J Neurol Neurosurg Psychiatry 77, 149-153.

5. Steffens DC, Potter GG, McQuoid DR, et al. (2007) Longitudinal magnetic resonance imaging vascular changes, apolipoprotein $\mathrm{E}$ genotype, and development of dementia in the neurocognitive outcomes of depression in the elderly study. Am J Geriatr Psychiatry 15, 839-849.

6. Longstreth WT Jr, Dulberg C, Manolio TA, et al. (2002) Incidence, manifestations, and predictors of brain infarcts defined by serial cranial magnetic resonance imaging in the elderly: the Cardiovascular Health Study. Stroke 33, 2376-2382.

7. Vermeer SE, Hollander M, van Dijk EJ, et al. (2003) Silent brain infarcts and white matter lesions increase stroke risk in the general population: the Rotterdam Scan Study. Stroke 34, 1126-1129.

8. Sachdev PS, Wen W, Christensen H, et al. (2005) White matter hyperintensities are related to physical disability and poor motor function. J Neurol Neurosurg Psychiatry 76, $362-367$

9. Corti MC, Baggio G, Sartori L, et al. (2007) White matter lesions and the risk of incident hip fracture in older persons: results from the Progetto Veneto Anziani study. Arch Intern Med 167, 1745-1751.

10. Bokura H, Kobayashi S, Yamaguchi S, et al. (2006) Silent brain infarction and subcortical white matter lesions increase the risk of stroke and mortality: a prospective cohort study. J Stroke Cerebrovasc Dis 15, 57-63.

11. Thomas AJ, O'Brien JT, Davis S, et al. (2002) Ischemic basis for deep white matter hyperintensities in major depression: a neuropathological study. Arch Gen Psychiatry 59, 785-792.

12. Payne ME, Anderson JJ \& Steffens DC (2008) Calcium and vitamin $\mathrm{D}$ intakes may be positively associated with brain lesions in depressed and nondepressed elders. Nutr Res 28, 285-292.

13. Bolland MJ, Barber PA, Doughty RN, et al. (2008) Vascular events in healthy older women receiving calcium supplementation: randomised controlled trial. BMJ 336, 262-266.

14. Pentti K, Tuppurainen MT, Honkanen R, et al. (2009) Use of calcium supplements and the risk of coronary heart disease in 52-62-year-old women: the Kuopio Osteoporosis Risk Factor and Prevention Study. Maturitas 63, 73-78.

15. Bolland MJ, Avenell A, Baron JA, et al. (2010) Effect of calcium supplements on risk of myocardial infarction and cardiovascular events: meta-analysis. BMJ 341, c3691.

16. Michaelsson K, Melhus H, Warensjo Lemming E, et al. (2013) Long term calcium intake and rates of all-cause and cardiovascular mortality: community based prospective longitudinal cohort study. BMJ 346, f228.

17. Bolland MJ, Grey A, Avenell A, et al. (2011) Calcium supplements with or without vitamin D and risk of cardiovascular events: reanalysis of the Women's Health Initiative limited access dataset and meta-analysis. BMJ 342, d2040.

18. Slinin Y, Blackwell T, Ishani A, et al. (2011) Serum calcium, phosphorus and cardiovascular events in post-menopausal women. Int J Cardiol 149, 335-340.

19. Li K, Kaaks R, Linseisen J, et al. (2012) Associations of dietary calcium intake and calcium supplementation with myocardial infarction and stroke risk and overall cardiovascular mortality in the Heidelberg cohort of the European Prospective Investigation into Cancer and Nutrition study (EPIC-Heidelberg). Heart 98, 920-925.

20. Karp HJ, Ketola ME \& Lamberg-Allardt CJ (2009) Acute effects of calcium carbonate, calcium citrate and potassium citrate on markers of calcium and bone metabolism in young women. Br J Nutr 102, 1341-1347.

21. Green JH, Booth C \& Bunning R (2003) Postprandial metabolic responses to milk enriched with milk calcium are different from responses to milk enriched with calcium carbonate. Asia Pac J Clin Nutr 12, 109-119.

22. Rubin MR, Rundek T, McMahon DJ, et al. (2007) Carotid artery plaque thickness is associated with increased serum calcium levels: the Northern Manhattan study. Atherosclerosis 194, 426-432.

23. Wang TK, Bolland MJ, van Pelt NC, et al. (2010) Relationships between vascular calcification, calcium metabolism, bone density, and fractures. J Bone Miner Res 25, 2501-2509.

24. Lind L, Skarfors E, Berglund L, et al. (1997) Serum calcium: a new, independent, prospective risk factor for myocardial infarction in middle-aged men followed for 18 years. $J$ Clin Epidemiol 50, 967-973.

25. Bailey RL, Dodd KW, Goldman JA, et al. (2010) Estimation of total usual calcium and vitamin D intakes in the United States. J Nutr 140, 817-822.

26. Steffens DC, Welsh-Bohmer KA, Burke JR, et al. (2004) Methodology and preliminary results from the neurocognitive outcomes of depression in the elderly study. J Geriatr Psychiatry Neurol 17, 202-211.

27. Frances AJ (editor) (1994) American Psychiatric Association: Diagnostic and Statistical Manual of Mental Disorders, Fourth Edition. Washington, DC: American Psychiatric Association.

28. Folstein MF, Folstein SE \& McHugh PR (1975) "Mini-mental state". A practical method for grading the cognitive state of patients for the clinician. J Psychiatr Res 12, 189-198.

29. Payne ME, Hybels CF, Bales CW, et al. (2006) Vascular nutritional correlates of late-life depression. Am J Geriatr Psychiatry 14, 787-795.

30. Block G (1992) Dietary assessment issues related to cancer for NHANES III. Vital Health Stat 4, 24-31.

31. Subar AF, Thompson FE, Kipnis V, et al. (2001) Comparative validation of the Block, Willett, and National Cancer Institute food frequency questionnaires: the Eating at America's Table Study. Am J Epidemiol 154, 1089-1099.

32. Payne ME, Fetzer DL, MacFall JR, et al. (2002) Development of a semi-automated method for quantification of MRI gray and white matter lesions in geriatric subjects. Psychiatry Res 115, 63-77.

33. Robins LN, Helzer JE, Croughan J, et al. (1981) National Institute of Mental Health Diagnostic Interview Schedule. Its history, characteristics, and validity. Arch Gen Psychiatry 38, 381-389.

34. Bos D, Ikram MA, Elias-Smale SE, et al. (2011) Calcification in major vessel beds relates to vascular brain disease. Arterioscler Thromb Vasc Biol 31, 2331-2337. 
35. Kim BJ, Lee SH, Kim CK, et al. (2011) Advanced coronary artery calcification and cerebral small vessel diseases in the healthy elderly. Circ J 75, 451-456.

36. Demuro A, Mina E, Kayed R, et al. (2005) Calcium dysregulation and membrane disruption as a ubiquitous neurotoxic mechanism of soluble amyloid oligomers. J Biol Chem 280, $17294-17300$.

37. de Leeuw FE, de Groot JC, Oudkerk M, et al. (2002) Hypertension and cerebral white matter lesions in a prospective cohort study. Brain 125, Pt 4, 765-772.

38. Artero S, Tiemeier H, Prins ND, et al. (2004) Neuroanatomical localisation and clinical correlates of white matter lesions in the elderly. I Neurol Neurosurg Psychiatry 75, 1304-1308.

39. Taylor WD, MacFall JR, Payne ME, et al. (2005) Greater MRI lesion volumes in elderly depressed subjects than in control subjects. Psychiatry Res 139, 1-7.

40. Vermeer SE, Den Heijer T, Koudstaal PJ, et al. (2003) Incidence and risk factors of silent brain infarcts in the population-based Rotterdam Scan Study. Stroke 34, 392-396.

41. Buell JS, Dawson-Hughes B, Scott TM, et al. (2010) 25-Hydroxyvitamin D, dementia, and cerebrovascular pathology in elders receiving home services. Neurology $\mathbf{7 4}, 18-26$. 